\title{
The capillarity equation at the nanoscale: gas bubbles in metals
}

\author{
A. Caro, D. Schwen, J. Hetherly, and E. Martinez* \\ Materials Science and Technology Division, Los Alamos National Laboratory, Los Alamos, NM 87544
}

(Dated: January 27, 2015)

\begin{abstract}
We investigate the modifications to the Young-Laplace capillarity equation needed to describe nanoscale gas bubbles embedded in metals, scale at which the finite width of the interface region can not be neglected. We focus in particular on the case of He in Fe. Using both, the concept of Tolman's length that provides a curvature dependence for the interface energy, and a new equation of state for He at the nanoscale that accounts for interface effects (see A. Caro et al. Appl. Phys. Lett. 103, 213115 (2013), we derive an expression to predict pressure, and from it density and the amount of $\mathrm{He}$ in nanoscale bubbles. We find that conditions for equilibrium are found for values of pressure or density at variance by a factor of $\sim 2$ compared to the traditional way of using the capillarity equation and a bulk He EOS.
\end{abstract}

\section{INTRODUCTION}

Capillarity, an old concept developed by Young and Laplace in the early $19^{\text {th }}$ century $[1,2]$, plays an essential role in many different fields of science and engineering. In the traditional definition, capillarity is the tendency of wetting liquids to be drawn into the confined space of a narrow tube. In a wider definition, it includes the phenomenon of enhanced pressure of a droplet due to the constrictive surface tension force. The concept in fact involves all phenomena in which two phases are separated by a curved interface, and as such it is of interest in biology, geology, physical chemistry, micro- and nanotechnology, and tribology, and for a diversity of applications such as electrospray, nano-fountain pen writing, capillarity bridges, and in natural sciences to understand certain animal locomotion and plants nutrition; for a review see van Honschoten[3].

Most of the studies in the literature, as well as the discussion that follows in this Section, consider the problem of capillarity between fluid phases. When two fluids, in mutual mechanical equilibrium, are separated by a spherical interface of radius $R$, the pressure of the fluid inside, $p^{\alpha}$, differs from that of the fluid outside, $p^{\beta}$. If the interface is assumed to be of zero thickness, the condition for mechanical equilibrium provides a simple relation between $p^{\alpha}$ and $p^{\beta}$, which is known as the Kelvin relation [4].

$$
\Delta P=p^{\alpha}-p^{\beta}=\frac{2 \gamma_{\infty}}{R}
$$

where $\gamma_{\infty}$ is the surface energy for a flat $(R \rightarrow \infty)$ surface. This method for treating surface tension is simple and useful but it is of an approximate nature from the molecular view point, for the structure of the fluid undergoes not a discontinuous but a progressive modification across the actual interface. When looked at the micro and nano scales, the effects of the finite width of

*Electronic address: caro@lanl.gov the interface. i.e. the existence of interfacial forces, or the molecular structure of matter, can not be neglected and need to be solved explicitly; by doing so fundamentally new phenomena appear, making capillarity at the nanoscale a vivid subject of research today.

As early as in 1949, based solely on thermodynamic arguments, Tolman showed that the Kelvin relation could be not valid for small fluid bubbles. He proved departures from the equation for the case of one-component two-phase systems of liquid bubbles in equilibrium with their vapor [5]. He introduced what since then is known as Tolman length $\delta$ that gives a correction term to the surface energy, namely:

$$
\gamma_{R} \simeq \gamma_{\infty} /\left(1+\frac{\delta}{R}\right)
$$

with $\delta$ in the range of intermolecular distances. Higher order terms in $1 / R^{n}$ are to be expected for small radii, and were discussed in Tolman's original paper.

A vast literature covers diverse aspects of the problem. Among them Ono and Kondo, and Rowlinson and Widow authored a review and a book respectively on the molecular theory of capillarity that are classics in the field $[6,7]$. As examples of recent work, we mention the contributions by Marchand et al.[8], and Style et al.[9-12] on the contact angle that a liquid drop makes on a soft substrate, which appears different from that predicted by Neumann's law [13], the complex scaling behavior of indentation of soft surfaces by adhesion forces [10] and the capillarity driven instabilities in soft solids[14-16].

Using computational modeling at the atomic scale, several papers look at the details of the interface region and evaluate pressures and densities for nanoscale bubbles or drops, mainly on Lennard-Jonnes model systems [17-24]. Of particular interest to us is the work by Thompson et al [25] who evaluates density profiles and put to test the concept of Tolman's length for the surface energy. They report density and pressure profiles that transition from the liquid to the vapor phases in a region (the interface) of the order of the interaction range of the potential, as expected from Tolman's theory.

Despite the abundant literature in this subject, we are not aware of a similar level of effort to describe gas bub- 
bles embedded in metals, which are expected to behave in a similar qualitative way, although with some differences, as we shall see below, given the fact that the solid containing the bubble is able to sustain shear. Such bubbles are a relevant microstructure feature in irradiated materials where noble gases such as $\mathrm{He}$, Xe, and $\mathrm{Kr}$ appear as a result of nuclear reactions. Their presence is usually a nuisance as they affect mechanical and thermal properties in dramatic ways [26, 27]. Chemically inert, these gases have a exceedingly low solubility in solids and a strong tendency to precipitate heterogeneously at defects such as dislocations, grain boundaries or precipitate interfaces.

To our knowledge, there has been no connection between the studies of nanoscale effects in bubbles, mostly in the field of colloids physics as briefly reviewed above, and the field of bubbles embedded in materials under irradiation. Support to this conclusion comes from the fact that the usual treatment of noble gas bubbles in materials, as for example given in the standard reference book by G. Was, 'Fundamentals of Radiation Materials Science' [28], uses Eq. 1 in combination with either an ideal gas or a van der Waals equation of state for the gas, to predict equilibrium and kinetic processes.

In this work we seek to establish this connection by bringing knowledge well established in colloidal physics to solid state physics and determine scaling effects for nanoscale He bubbles in Fe, aiming at providing a quantitative evaluation of the departure from the YoungLaplace equation (1) as we enter the nanoscale, and establishing the conditions under which a bubble is said to be in equilibrium with its environment.

Motivation for our study comes from our recent work on the structure of these nanoscale bubbles [29]. There we evaluate the pressure inside a He bubble and show that, in contrast to the common assumption for macroscale bubbles, that pressure (the trace of the stress tensor) is constant, for He bubbles at the nanoscale this picture is no longer valid. $P$ and density $\rho$ can no longer be defined as global quantities related by an equation of state, EOS, for the fluid, but they become functions of position, because the bubble develops a core-shell structure originated in the atomic scale dimensions of the interface, in a similar way as reported by Thompson for Lennard Jones fluids [25]. The range of the metal-gas interaction defines the width $\delta$ of the interface, which for the He-Fe system studied, is $\delta \sim 0.4 \mathrm{~nm}$, implying that bubbles in the rage of $1 \mathrm{~nm}$ diameter are almost entirely affected by this interface effect, creating a radial pressure profile that within a distance $\delta$ from the interface, is not constant in the fluid, nor zero in the metal, as the theory of the Eshelby inclusion, which is valid beyond the interface, would predict [30].

The paper is organized as follows: we first calculate via direct computer simulations, the pressure, density and number of particles in equilibrium bubbles. Then we apply the Tolman's theory to fit these results with an R-dependent surface energy. We then develop an EOS for nanoscale bubbles, and finally, we compare our predictions with the results of simulations. A discussion sections finalizes the work.

\section{RESULTS}

\section{A. Equilibrium bubbles and the capillarity equation}

A usual way to estimate the amount of gas in a bubble embedded a metal is to assume mechanical and thermodynamic equilibrium, i.e., the gas pressure equals the capillarity force $P=2 \gamma / R$, Eq. (1) and the chemical potential for vacancies far form the bubble and at its surface are equal; under this condition there is no driving force for vacancies to be preferentially emitted or absorbed by the bubble. The amount of gas in the bubble can then be estimated using Eq. 1 just from its size, the surface energy of the host matrix, and an EOS for the fluid relating $P, T$ and density.

The first question we address is a quantification of the discrepancy between the prediction of Eq. 1 and the result of an atomic scale calculation of equilibrium bubbles. We study the case of He in Fe using empirical potentials and molecular dynamics. For details on the simulations see Ref. [29]. A bubble in (mechanical) equilibrium is a bubble containing exactly the amount of gas that produces no perturbation to the elastic fields (stress and strain) of the surrounding metal atoms, at a distance larger that the interface width of $\sim 0.4 \mathrm{~nm}$ mentioned above. To find this condition we use a simulation cell consisting of a spherical Fe crystal with five concentric shell regions, all but the inner-most of thickness equal to the cut-off of the potential $r_{c u t}$. The inner-most region is the bubble of varying radius, where He sits.

The sample is created with the lattice constant of $\mathrm{Fe}$ at the chosen target temperature. It is embedded in vacuum. Atoms in the outermost shell of this iron ball are frozen in place. Traveling inwards, this shell is followed by another shell with moving Fe atoms that forms a buffer zone between the frozen atoms and the so called integration zone, the shell of moving Fe atoms where stress calculations are performed. The integration region is followed by a second buffer region which screens from the interactions with He gas atoms. The innermost shell contains the He gas in a spherical cavity.

The precise characterization of the elastic fields in an spherical domain with frozen boundary conditions can be found in Ref.[31], but here we are only interested in equilibrium bubbles, situation where these fields are zero, which greatly simplifies the analysis. If instead of the gas in the cavity, we place perfect crystal Fe, the pressure and all the elements of the stress tensor in the integration region become zero, within numerical accuracy and thermal noise. Now, placing in the inner zone gas atoms at some varying density, the condition of zero pressure in the integration zone will be obtained for a gas den- 


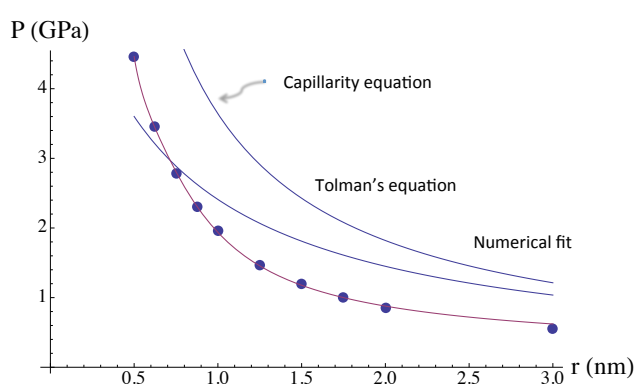

FIG. 1: Pressure vs radius for equilibrium He bubbles in $\mathrm{Fe}$ from three different approaches: the capillarity equation (Eq. (1)), the first order Tolman's expression, equations (1) and (2), and an empirical expression fitted to molecular dynamics results, equation (5). The results form computer simulations show a weak temperature dependence.

sity corresponding to equilibrium bubbles. A number of simulations $(\sim 5000)$ were performed at combinations of multiple temperatures $T$, bubble radii $R$ and densities $\rho$. Pressure is determined as the average of per-atom pressures, which in turn are calculated using the peratom stress-times-volume tensor traces and the per-atom Voronoi volumes. In a post processing step the pressures per atom are averaged temporally, yielding a pressure value for He in the bubble $P(R, T, \rho)$ and for Fe atoms in the integration region $p^{\prime}(R, T, \rho)$. For each set $R, T$ we next determine via interpolation the value of $\rho$ (and the number of He atoms $N_{H e}$ ) at which $p^{\prime}$ in the integration region is zero. This density is the equilibrium He density $\rho_{e q}(R, T)$.

A comment here is needed with respect to the use of Voronoi volumes to define stress tensors. This quantity fulfills some basic requirements for a proper definition of atomic volume, namely, no fraction of space is left unassigned or counted more than once, i. e. there is no overlap of polyhedra, which warrants that the sums of all atomic volumes gives the total volume. However, it fails to properly assign space to atoms in situations of strong heterogeneity such as free surfaces or bubbles at low density. In this last case, the Voronoi volume of the gas is not defined as the space the gas atoms can occupy, as can be shown with the following argument. Suppose a solid with a cavity of radios $\mathrm{R}$, and suppose a single He atoms sits inside it; for simplicity, let's assume it is at its center. The density should be 1 atom $/ \frac{4}{3} \pi R^{3}$, while the Voronoi volume would be $\sim 1 / 8$ of the volume of the cavity because the Voronoi polyhedra is constructed with the bisector planes between He and metal atoms. It then gives a density $\sim 8$ times higher than real. In summary, for low densities, the density and stresses are overestimated. For the purpose of our study, densities are always close to unity in He/Vac units, which warrants that the Voronoi polyhedron is a proper evaluation of atomic volumes

Another different and important limitation comes from the commonly used definition of the stress tensor of a set of atoms interacting via a potential by Basinki etal. [32] as is implemented in the MD code Lammps [33] used for this work, which is not consistent with conservation of local momentum. Cormier etal. [34] discuss this issue and derive an expression that does not suffer from this limitation. Their expression shows correct asymptotic behavior for large volumes, and some departures from the Basinki etal. expression for volumes corresponding to $\sim 2$ lattice spacings, highlighting the limited accuracy of the Basinki approach.

While we are aware of both these limitations, namely incorrect density measurement at low density and inaccurate stress value at the atomic level, our use of Basinki etal. stress tensor via Voronoi volume calculations is just one of the approximations we adopt to obtain a computationally simple way to get values of the stress; the other important approximation is the use of an average of stress tensor per He atom in an heterogeneous bubble to get a single quantity for the bubble to be used in an EOS. Finally, our results are consistent with this definition of atomic volume in the sense that all densities and stresses are defined via the volume of He atoms as defined by their Voronoi volume and not by the radius of the cavity cut out from the metal.

Figs 1 to 3 show plots of $P_{e q}, \rho_{e q}$ and $N_{H e}$ versus bubble radius for equilibrium bubbles at different temperatures as obtained from the computer simulations. Fig. 1 also shows Eq. 1 for Fe with $\gamma=1.82 \mathrm{GPa}$ nm, value obtained as the average for this potential for a flat surfaces along three different ortientations $\left(\gamma_{(100)}=1.74\right.$, $\left.\gamma_{(110)}=1.62, \gamma_{(111)}=2.10 \mathrm{GPa} \mathrm{nm}\right)$. The discrepancy between the prediction of the capillarity equation and the MD results is notorious: for nanoscale bubbles with radius below $\sim 3 \mathrm{~nm}$ the actual equilibrium pressure is about a factor of 2 below the Young-Laplace prediction. The temperature dependence of the MD data in Fig. 1 is weak, as is to be expected from Eq. 1, where the only T-dependence comes from $\gamma$. The dispersion of the MD data at large bubble radii reflects numerical inaccuracy.

A discrepancy in pressure implies a discrepancy in density and in the number of He atoms in equilibrium bubbles. From Eq. 1 and a He EOS it is possible to obtain the equilibrium He density predicted by the capillarity equation. Using the EOS for bulk He that we report below, Eq. (10), we plot in Fig. 2 the values obtained for different $T$ according to Eq. (1), together with the results of the computer simulation where density is simply the number of He atoms divided by the sum of the Voronoi volumes of all He atoms in the bubble. Once again, the discrepancy is notorious, with the actual density well below the capillarity equation prediction.

Finally, with the He density and the bubble radius, we can evaluate the capillarity equation prediction for the number of He atoms in equilibrium bubbles at dif- 


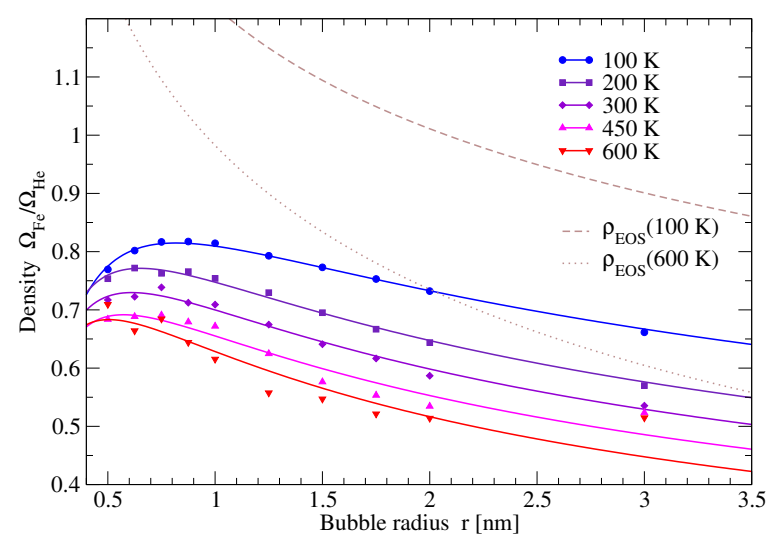

FIG. 2: Density vs radius for equilibrium He bubbles in Fe at different temperatures from molecular dynamics results. Also shown is the density that results from Eq. 1 and the bulk He EOS.

ferent radii and temperatures, which we show in Fig. 3, together with the MD results. This figure summarizes the first main result of this work, that says that if the capillarity equation is used to estimate the amount of $\mathrm{He}$ in equilibrium nanoscale bubbles in Fe, an error of a factor of 2 or more is made for bubbles in the range of $1 \mathrm{~nm}$ in diameter. This result is probably qualitatively valid for all fluid bubbles in solids, as it comes from the finite width of the interface region, determined by the gas-matrix interaction range.

We point out that in this work we use the word fluid to characterize $\mathrm{He}$ in bubbles because at the temperatures and pressures of interest, we are always well above the critical point for $\mathrm{He}\left(T_{c}=5.19 \mathrm{~K}\right.$ and $P_{c}=0.227 \mathrm{MPa}$, where liquid and gas phases become indistinguishable.

The inadequacy of the capillarity equation to predict equilibrium conditions also affects non-equilibrium situations. Suppose we are interested on determining the rate of return to equilibrium of an over- or under- pressurized bubble under condition of thermal annealing. A simple rate equation for this process would read,

$$
\frac{d R}{d t}=\frac{\Omega D_{v}}{R}\left[C_{v}^{e}-C_{v}^{c}\right]
$$

where $R$ is the bubble size, $\Omega$ is the volume of an atom, $D_{v}$ is the vacancy diffusion coefficient, $C_{v}$ is the vacancy concentration in bulk, and $C_{v}^{c}$ is the vacancy concentration at the surface of the bubble [28]. The first term on the r.h.s. represents the contribution to growth rate via vacancy capture, the second represents the evaporation of vacancies and depends on their concentration at the surface of the bubble, a quantity that depends on the departure from the equilibrium condition expressed by Eq. 1 , and is given by,

$$
C_{v}^{c}=C_{v}^{e} e^{\left[-\mu_{v} / k T\right]}
$$

with $C_{v}^{e}=e^{\left[\epsilon_{v}^{f} / k T\right]}$ and $\mu_{v}=\Omega\left(\frac{2 \gamma}{R}-P\right)$, where $\gamma, P, \Omega$, and $R$ were already defined and $k$ the Boltzmann constant, $T$ the temperature, $C_{v}^{e}$ the thermal equilibrium

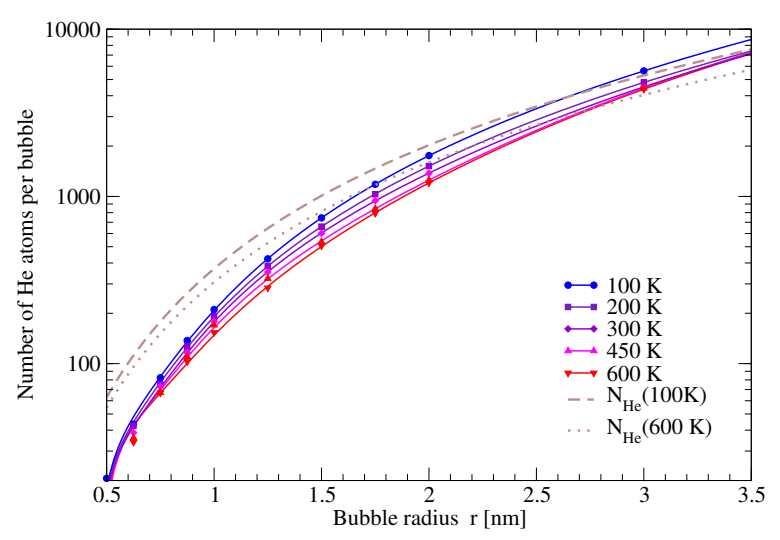

FIG. 3: Number of atoms in equilibrium He bubbles in Fe at different temperatures. Also shown is the number of atoms obtained from the bulk He EOS density, Eq. (10) for $\mathrm{R} \rightarrow \infty$, and $\mathrm{P}$ given by Eq. (1).

vacancy concentration, $\epsilon_{v}^{f}$ the formation energy of a vacancy in bulk, and $\mu_{v}$ the chemical potential of a vacancy at the interface. We see then that the inadequacy of the capillarity equation to predict the equilibrium pressure has implications not only in predicting how much gas is in an equilibrium bubble but also on predictions of rate processes.

The reasons for the discrepancy between the MD results and Eq. 1 has two origins. One is the use of the capillarity equation itself because, as we discussed in the Introduction, Tolman's theory indicates that $\gamma$ depends on radius. The other is the use of the bulk He EOS that is equivalent to the assumption that pressure in a nanoscale confined fluid still follows Pascal law, i.e. is constant within the fluid, with no influence of the surrounding metal, and therefore that a bulk He EOS can be used to relate pressure to density. This assumption also breaks at the nanoscale where the width of the interface, i.e. the region where metal-He interaction plays a role, is of the same dimensions of the bubble itself, as we discussed in detail in [29]. In what follows, we treat these two sources of discrepancy separately.

\section{B. Tolman's length}

As discussed in the Introduction, Eq. 2 represents a first order correction to the surface energy $\gamma_{R}$ of fluids. In Fig 1 we also show Eq. 1 including that correction. The value for the Tolman's length that best fits the data is $\delta=0.26 \mathrm{~nm}$, comparable, but significantly smaller that the range of the Metal-He interaction of $0.4 \mathrm{~nm}$. The fit is poor, indicating that, while the correction goes in the right direction of decreasing the pressure, higher order terms are needed. In the original paper by Tolman, the higher order corrections are not given explicitly, as they come from an integral that has no analytic solution. We follow here a different approach, trying an empirical expression that adds higher order $1 / R^{n}$ terms, namely: 


$$
\gamma_{R} \simeq \gamma_{\infty} /\left(1+\frac{\delta_{1}}{R}+\frac{\delta_{2}}{R^{2}}+\frac{\delta_{3}}{R^{3}}+\frac{\delta_{4}}{R^{4}}\right)
$$

where $\delta_{i}$ are fitting parameters. As shown in Fig 1 , the fit obtained with the values $\delta_{1}=4.97 \mathrm{~nm}, \delta_{2}=-7.39 \mathrm{~nm}^{2}$, $\delta_{3}=4.21 \mathrm{~nm}^{3}$, and $\delta_{4}=-0.812 \mathrm{~nm}^{4}$, is excellent. We do not attempt to give an interpretation to these values since Eq. 5 is now totally empirical. It suffices to say that Eq. 5 brings the main concept of Tolman's length in that the surface energy depends on curvature radius, it has the right asymptotic behavior for $R \rightarrow \infty$, and fits the MD data very well in the region under study.

We stress here the point that Tolman's treatment is for fluids while our study is for a fluid embedded in a crystal: if we remove the fluid, the cavity is still there, because the solid does not collapse, in clear contrast with the case of a a bubble embedded in a fluid.

\section{EOS for $\mathrm{He}$ in bubbles}

Having determined the pressure for equilibrium bubbles, we need now to calculate the density; these two quantities are related by an EOS. As we showed in [29] the gas pressure in a nanoscale bubble is a position dependent quantity for $\mathrm{He}$ in $\mathrm{Fe}$, a fact that is known for fluids in general [25].

The conclusion that the EOS for He in bubbles is different from the EOS of bulk He can be clearly deduced from inspection of the data in Figs. 1 and 2. The pressure in the first figure and the density in the second are obtained by direct measurements of the simulation data; the relation between them is an EOS. Curiously, this EOS is significantly different from the bulk He EOS, in that it depends on the bubble radius. As an example, if we read from Fig. 1,

$$
P_{e q}(r=1 n m, T=450 \mathrm{~K})=1.91 \mathrm{GPa}
$$

and from Fig. 2

$$
\rho_{e q}(r=1 \mathrm{~nm}, \mathrm{~T}=450 \mathrm{~K})=0.67 \mathrm{He} / \mathrm{Vac}
$$

we get an EOS that gives

$$
P_{\text {bubble }}(\rho=0.67 \mathrm{He} / \mathrm{Vac}, \mathrm{T}=450 \mathrm{~K})=1.91 \mathrm{GPa}
$$

while for bulk $\mathrm{He}(\mathrm{R} \rightarrow \infty) P$ as given by the EOS that we report later in this article, Eq. 10, is

$$
P_{\text {bulk }}(\rho=0.67 \mathrm{He} / v a c, T=450 \mathrm{~K})=1.08 \mathrm{GPa} .
$$

The discrepancy between the two values of pressures suggests that the EOS of He in nanoscale bubbles depends on its radius.

To establish an EOS for He in bubbles with an explicit dependence on radius that could be used in Eqs. 1 or 4 to predict equilibrium or rate processes, we perform

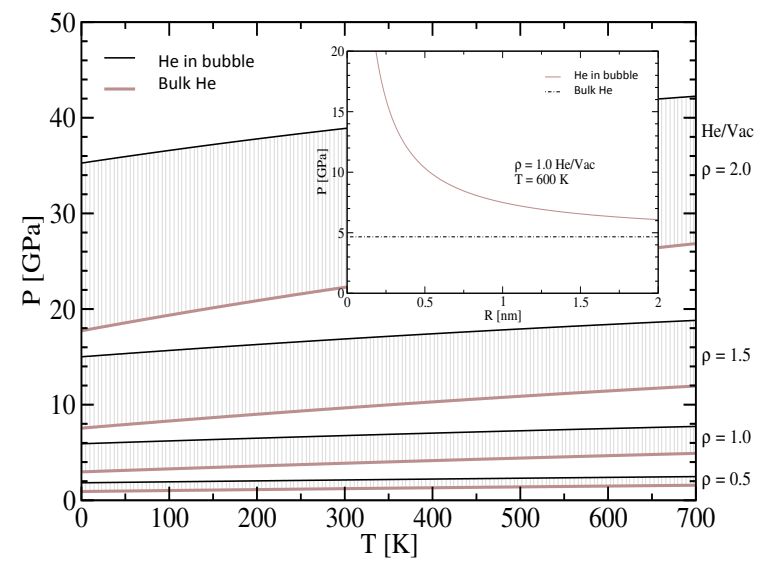

FIG. 4: Cuts of the bubble EOS $P(\rho=$ $0.5,1,1.5,2 \mathrm{He} / \mathrm{Vac}, \mathrm{T}, r=1 \mathrm{~nm})$ showing $\mathrm{P}$ vs $\mathrm{T}$ at different densities. Also shown are the curves corresponding to bulk He EOS. The shaded area between the curves represent the increase in pressure induced by interface effects. Inset: $\mathrm{EOS}$ cut $P(\rho=1 \mathrm{He} / \mathrm{Vac}, \mathrm{T}=450 \mathrm{~K}, r)$ showing $\mathrm{P}$ vs r. Also shown is the pressure for bulk He at the same density and temperature.

a systematic evaluation of the relation between R- $\rho$-T-P based on the previously defined pressure average. The procedure is the same used to determine the equilibrium conditions, this time enlarging the domain of the variable density to cover a region between 0.3 and 2 times the number of metal atoms removed (the unit of density used throughout this work is $\mathrm{He} /$ matrix atom vacancy). The sample is equilibrated and then the average pressure and density of the He atoms are measured. The final result for each bubble is its radius, density, temperature and pressure. The values for several hundred $(R, \rho, T, P)$ points represent the raw data for the EOS. A non-linear fitting procedure determines the parameters in the expression we use for the EOS, namely:

$$
\begin{aligned}
P(\rho, T, R)= & \left(1+\frac{b_{0}+b_{1} T+b_{2} T^{2}}{R}+\frac{c_{0}+c_{1} T+c_{2} T^{2}}{R^{2}}+\right. \\
& \left.\frac{d_{0}+d_{1} T+d_{2} T^{2}}{R^{3}}+\frac{e_{0}+e_{1} T+e_{2} T^{2}}{R^{4}}\right) \times \\
& \times\left(a_{0}(\rho)+a_{1}(\rho) T+a_{2}(\rho) T^{2}\right)
\end{aligned}
$$

with

$$
\begin{aligned}
& a_{0}(\rho)=\exp \left(f_{0}+f_{1} \rho+f_{2} \rho^{2}+f_{3} \rho^{3}+f_{4} \rho^{4}\right)-a_{0}(0) \\
& a_{1}(\rho)=\exp \left(g_{0}+g_{1} \rho+g_{2} \rho^{2}+g_{3} \rho^{3}+g_{4} \rho^{4}\right)-a_{1}(0) \\
& a_{2}(\rho)=-\exp \left(h_{0}+h_{1} \rho+h_{2} \rho^{2}+h_{3} \rho^{3}+h_{4} \rho^{4}\right)-a_{2}(0)
\end{aligned}
$$

Despite the large number of parameters, this equation is only valid in a reduced region of the parameter space, namely for $0.5 \mathrm{~nm}<R<3 \mathrm{~nm}, 0.3 \mathrm{He} / \mathrm{Vac}<\rho<$ $2 \mathrm{He} / \mathrm{Vac}$, and $100 \mathrm{~K}<\mathrm{T}<700 \mathrm{~K}$. Eq. 10 is composed 
TABLE I: Parameters for the EOS of He, Eqs.( 10) and (11).

\begin{tabular}{|c|c|c|}
\hline Parameter & Fit & Standard error \\
\hline$b_{0}$ & 6.97 & 0.88 \\
\hline$b_{1}$ & $2.14 \times 10^{-5}$ & $6.4 \times 10^{-6}$ \\
\hline$b_{2}$ & -0.0268 & 0.0052 \\
\hline$c_{0}$ & -0.000227204 & 0.000048 \\
\hline$c_{1}$ & $-1.56 \times 10^{-5}$ & $4.7 \times 10^{-6}$ \\
\hline$c_{2}$ & 0.0195 & 0.0038 \\
\hline$d_{0}$ & -5.21 & 065 \\
\hline$d_{1}$ & -0.000189015 & 0.00002 \\
\hline$d_{2}$ & -0.000189015 & 0.00002 \\
\hline$e_{0}$ & 1.25 & 0.15 \\
\hline$e_{1}$ & $3.65 \times 10^{-6}$ & $1.1 \times 10^{-6}$ \\
\hline$e_{2}$ & -0.00453 & 0.0009 \\
\hline$f_{0}$ & -7.96088 & 0.23 \\
\hline$f_{1}$ & 14.6389 & 0.65 \\
\hline$f_{2}$ & -9.21756 & 0.66 \\
\hline$f_{3}$ & 3.27782 & 0.29 \\
\hline$f_{4}$ & -0.477841 & 0.048 \\
\hline$g_{0}$ & -8.79056 & 0.20 \\
\hline$g_{1}$ & 5.39224 & 0.69 \\
\hline$g 2$ & -2.4015 & 0.87 \\
\hline$g_{3}$ & 0.47301 & 0.46 \\
\hline$g_{4}$ & -0.029434 & 0.086 \\
\hline$h_{0}$ & -18.29 & 0.76 \\
\hline$h_{1}$ & 5.21 & 2.53 \\
\hline$h_{2}$ & -0.676 & 3.0 \\
\hline$h_{3}$ & -0.80 & 1.5 \\
\hline$h_{4}$ & 0.23 & 0.28 \\
\hline
\end{tabular}

of two parts, the first parenthesis on the r.h.s contains the radial dependence and the second represents the EOS for bulk He in the limit $R \rightarrow \infty$.

Two particular cuts of the EOS are presented in Fig. 4 , namely, the inset shows the average pressure of a bubble at $\mathrm{T}=600 \mathrm{~K}$ and $\rho=1 \mathrm{He} / \mathrm{Vac}$ at varying radius in the range $0-2 \mathrm{~nm}$; also in the inset the pressure of bulk $\mathrm{He}$ at that same density and temperature is indicated as a horizontal line. As expected, there is a significant difference at the same density between pressure in bulk $\mathrm{He}$ and pressure in bubbles for radius in the nm range. The main figure shows the EOS cut $P$ vs. $T$ for $1 \mathrm{~nm}$ radius bubbles at different densities. Shaded areas between the curves help visualize the interface and curvature effects on pressure.

\section{Predictions for equilibrium conditions}

It would be of interest for practical applications to invert the EOS Eq. 11 in order to be able to predict density and number of $\mathrm{He}$ atoms in equilibrium bub-

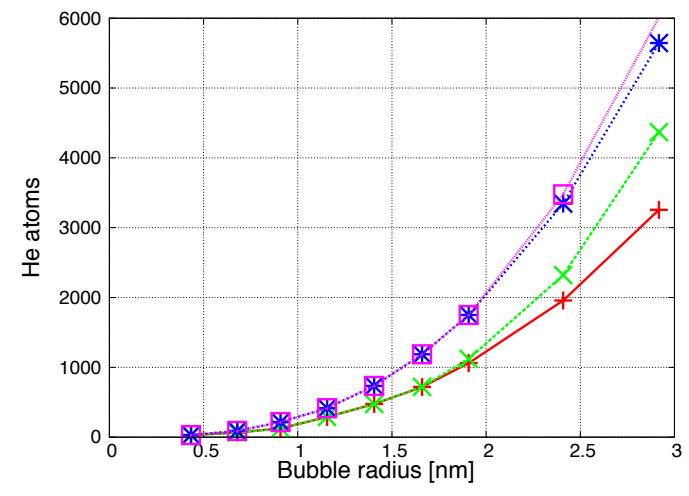

FIG. 5: Number of Helium atoms in equilibrium bubbles of different radii, at $\mathrm{T}=100$ (stars and squares symbols) and 700 $\mathrm{K}$ (pluses and crosses symbols) showing the predictions of Eq. 12 (pluses and stars symbols), together with the MD results of Fig. 3 (crosses and squares symbols).

bles of different radius at different Ts. Since Eq. 11 is not analytically invertible, we follow a numerical procedure to determine the density $\rho$ that satisfy the equation $P\left(\rho_{\text {equal }}, T, R\right)=P_{\text {Tolman }}(R)$. Using this density, the number of He atoms in an equilibrium bubble of radius $\mathrm{R}$ at temperature $\mathrm{T}$ is simply given by,

$$
n_{\text {equil }}(T, R)=\rho_{\text {equil }}(T, R) \frac{4}{3 \Omega} \pi R^{3}
$$

Fig. 5 shows Eq. 12 at two temperatures, namely 100 and $700 \mathrm{~K}$, together with the results from the MD simulations reported in Fig. 3, represented this time on a linear scale. The numerical predictions obtained by inversion of the EOS are in good agreement with the direct computer simulations results, although the agreement is less satisfactory at large radius, reflecting limitations in the accuracy of the EOS, Eq. (10). This figure represents the second main result of this work, a prediction, via Eqs. (5), (10) and (12) of the amount of He in equilibrium bubbles in Fe.

\section{DISCUSSION AND CONCLUSIONS}

During the process of revising this manuscript, we became aware of a recent similar work by Stoller and Osetsky [36] in which they reformulate an EOS for He in bubbles in terms of a modified hard spheres EOS. Using their three-body interatomic potential for the He-Fe interactions, they determine the conditions for mechanical equilibrium in a way similar to ours, and map the pressurevolume relationship for the bubbles at a range of temperatures. These atomistic results are compared to an existing equation of state and a modification is proposed for bubbles with small radii. A detailed comparison between both works suggest that the basic conclu- 
sions regarding what densities correspond to equilibrium bubbles are similar, as is the strategy of defining volumeaveraged densities and pressures. Important differences though are the facts that they do not address neither the problem of the surface energy dependence on radius, our modified Tolman's equation, nor the need of a proper definition of atomic volume, which enters the definition of pressure. Results from both works become closer as the radius of the bubble is above $\sim 2 \mathrm{~nm}$. Regarding the surface energy, they find that the equilibrium pressure follows approximately the capillarity equation when the surface energy used is the actual value determined by MD for bubbles of $2 \mathrm{~nm}$, i. e. another important approximation that is not the value for a flat surface, nor a value that depends on radius.

Importantly, the comparison of results from Stoller etal. and us to the experimental measurements of helium density by $\mathrm{Wu}$ etal. for a $1.3 \mathrm{~nm}$ bubble that lead to a He/vacancy ratio 0.6 [37] , and earlier measurements by Frechard etal. for bubbles between 2 and $5 \mathrm{~nm}$ for which the $\mathrm{He} /$ vacancy ratio was in the range of $0.25 \sim$ $0.85[38]$ indicate that both theoretical predictions are in equivalent reasonable agreement with experiments. For a better theoretical accuracy, only a comparative study of potentials using the same definitions for volumes, pressures, and surface energies may assess the importance of potentials in determining these properties.

In summary, in this work we used computer simulations to determine the limits of validity of the capillarity equation (1) for bubbles at the nanoscale. We first evaluate the relation between pressure, density and number of particles as a function of temperature and bubble radius for equilibrium bubbles, Figs. 1-3. To obtain these results we used average pressure, since this variable becomes position dependent inside the bubble, as we discussed in [29].Then we compare these results with the prediction of the capillarity equation (1); we find that for radii in the range of a few $\mathrm{nm}$ the validity of that equation is highly compromised, see Fig 1.

We then applied the Tolman's correction to the capillarity equation, Eq. 2, and find that while the correction goes in the right direction, it is insufficient to reproduce the results of the MD simulations. We then define an empirical expression for the surface energy variation with radius, Eq. 5. This Eq. predicts quite accurately the av- erage pressure in equilibrium He bubbles in $\mathrm{Fe}$ as a function of radius and temperature. However, to determine the density or the number of He atoms in the equilibrium bubbles, we need to relate pressure to density, an EOS. Analysis of the MD results shows that this relation depends on $\mathrm{R}$, and therefore a new He EOS needs to be defined.

By running a large number of cases for different radius, temperatures and densities we fit a numerical expression for the new EOS, Eq. 10. Numerical inversion of this Eq. gives the density as a function of pressure, and from it we get the number of He atoms in equilibrium bubbles, Eq. 12.

These last two equations allow the determination of the number of He atoms that make a bubble to be in thermodynamic equilibrium with the matrix.

A practical consequence of the inaccuracy of the capillarity equation at the nanoscale is that the number of $\mathrm{He}$ atoms that make a bubble embedded in $\mathrm{Fe}$ to be in equilibrium with its environment is predicted to be twice the value obtained by a direct computer simulation. The reasons for this failure is that at the nanoscale, the structure of the Fe-He interface can not be neglected, introducing more complexity. We capture this complexity by defining both a new expression for the surface energy versus radius, and a new EOS for He in bubbles, that depends on the bubble radius. While figures 1-3 allow a rapid determination of equilibrium conditions for bubbles in Fe, a full analytic description is provided by equations that are numerically fitted to computational results.

This study reveals another nanoscale effects on capillarity that, similar to the cases discussed in the introduction for drops at the interface of two other materials, shows how rich an old problem becomes when studied at the nanoscale.

\section{Acknowledgments}

This work was performed by the Center for Materials at Irradiation and Mechanical Extremes, an Energy Frontier Research Center funded by the U.S. Department of Energy (Award Number 2008LANL1026) at Los Alamos National Laboratory.
[1] T. Young, Philos. Trans. R. Soc. London, 1805, 95, 6587.

[2] P. S. Laplace, OEuvres completes de Laplace, IV, Supplement au livre $\mathrm{X}$ du traite de la mecanique celeste, $\mathrm{p}$. 394, 2e supplement au livre X, 1805, p. 419, ch. I.

[3] Joost W. van Honschoten, Nataliya Brunets and Niels R. Tas. Chem. Soc. Rev., 2010, 39, 10961114.

[4] W. Thomson, Phil. Mag. 42, 448, (1871).

[5] R. C. Tolman, J. Chem Phys. 17, 333 (1949).

[6] S Ono and S. Kondo. Molecular theory of surface tension in liquids. H. S. Green at al. Structure of Liquids.
Springer Verlag Berlin 1960.

[7] J. S. Rowlinson and B. Widom, Molecular Theory of Capillarity, Clarendom Press 1982.

[8] Antonin Marchand, Siddhartha Das, Jacco H. Snoeijer, and Bruno Andreotti. Phys. Rev. Lett. 108, 094301 (2012)

[9] Robert W. Style, Rostislav Boltyanskiy, Yonglu Che, J. S. Wettlaufer, Larry A. Wilen, and Eric R. Dufresne. Phys. Rev. Lett. 110, 066103 (2013).

[10] Robert W. Style, Callen Hyland, Rostislav Boltyanskiy, 
John S. Wettlaufer and Eric R. Dufresne. Nature Communications. DOI: 10.1038/ncomms3728 (2013).

[11] R. W. Style and E. R. Dufresne. Soft Matter, 2012, 8, 7177.

[12] Robert W. Style, Yonglu Che, Su Ji Park, Byung Mook Weon, Jung Ho Je, Callen Hyland, Guy K. German, Michael P. Power, Larry A. Wilen, John S. Wettlaufer, and Eric R. Dufresne. PNAS (2013) 1101254112544

[13] Franz Neumann with A. Wangerin, ed., Vorlesungen über die Theorie der Capillarität [Lectures on the theory of capillarity] (Leipzig, Germany: B. G. Teubner, 1894).

[14] Rohini Gupta , Gloria K. Olivier and Joelle Frechette. Langmuir, 2010, 26 (14), pp 1194611950

[15] C. Py, P. Reverdy, L. Doppler, J. Bico, B. Roman and C. N. Baroud, Phys. Rev. Lett., 2007, 98, 156103.

[16] Serge Mora, Ty Phou, Jean-Marc Fromental, Len M. Pismen and Yves Pomeau. Phys. Rev. Lett. 105, 214301 (2010).

[17] Benjamin J. Block, Subir K. Das, Martin Oettel, Peter Virnau, and Kurt Binder. The Journal of Chemical Physics 133, 154702 (2010).

[18] A. E. van Giessen, E. M. Blokhuis, and Dirk Jan Bukman. The Journal of Chemical Physics 108, 1148 (1998).

[19] E. M. Blokhuis and D. Bedeaux. The Journal of Chemical Physics 97, 3576 (1992).

[20] H. M. Lu and Q. Jiang. Langmuir 2005, 21, 779-781.

[21] K. Koga, X. C. Zeng, and A. K. Shchekin. The Journal of Chemical Physics 109, 4063 (1998).

[22] V. Simmons and J. B. Hubbard. The Journal of Chemical Physics 120, 2893 (2004).

[23] M. J. Haye and C. Bruin. The Journal of Chemical Physics 100, 556 (1994)

[24] J F Lutsko, J Laidet and P Grosfils. J. Phys.: Condens. Matter 22 (2010) 035101

[25] S. M. Thompson, K. E. Gubbins, J. P. R. B. Walton,
R. A. R. Chantry, and J. S. Rowlinson. The Journal of Chemical Physics 81, 530 (1984).

[26] Odette, G. R.; Alinger, M. J.; Wirth, B. D. Annual Review of Materials Research Book Series: Annual Review of Materials Research 38 471-503 (2008).

[27] Hu, Shenyang; Henager, Charles H., Jr.; Heinisch, Howard L.; et al. Jr. Nuc. Materails 392 292-300 (2009).

[28] Was, Gary S. Fundamentals of Radiation Materials Science. Springer (2007) ISBN 978-3-540-49472-0.

[29] A. Caro, D. Schwen, and E. Martinez. Applied Physics Letters 103, 213115 (2013).

[30] ESHELBY, JD. Proc. of the Royal Soc. of London Series A-Mathematical and Phys. Sci. 241 376-396 (1957).

[31] Shaofan Li,Roger A. Sauer and Gang Wang. Journal of Applied Mechanics (2007) 74 p 771.

[32] Z. S. Basinski, M. S. Duesbery, and R. Taylor, Can. J. Phys. 49, 2160 (1971).

[33] Plimpton S. Journal of Computational Physics 117, 1 (1995).

[34] J. Cormier, J. M. Rickman, T. J. Delph. Journal of Appl. Physics 89, 99 (2001).

[35] A. Caro, J. Hetherly, A. Stukowski, M. Caro, E. Martinez, S. Srivilliputhur, L. Zepeda-Ruiz, M. Nastasi. Journal of Nuclear Materials 418 (2011) 261268.

[36] R.E. Stoller and Yu.N. Osetsky. Journal of Nuclear Materials 455 (2014) 258262

[37] Y. Wu, G.R. Odette, T. Yamamoto, J. Ciston, P. Hosemann, An Electron Energy Loss Spectroscopy Study of Helium Bubbles in Nanostructured Ferritic Alloys, Fusion Reactor Materials, Semiannual Progress Report DOE/ER-0313/54, Oak Ridge National Laboratory (2013) 173179

[38] S. Frchard, M. Walls, M. Kociak, J.P. Chevalier, J. Henry, D. Gorse, J. Nucl. Mater. 393 (2009) 102. 